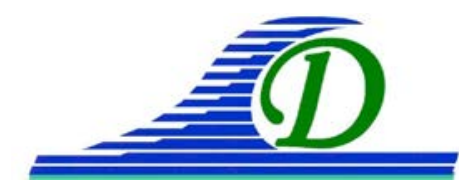

XIII ${ }^{\text {èmes }}$ Journées Nationales Génie Côtier - Génie Civil Dunkerque, 2-4 juillet 2014

DOI:10.5150/jngcgc.2014.109 (C) Editions Paralia CFL

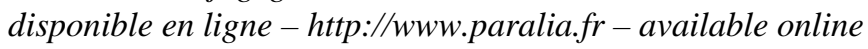

\title{
Cinq ans de valorisation des sédiments contaminés extraits du Port de Dunkerque (France)
}

\section{Sofie HERMAN ${ }^{1}$, Daphné GLASER ${ }^{1}$, Alain PIETERS ${ }^{1}$, Pascal GREGOIRE ${ }^{2}$, Christophe PRIEZ $^{3}$, Didier DESMOULIN ${ }^{4}$, David GUGLIELMETTI ${ }^{5}$}

1. Envisan, membre du Jan De Nul Group, 12 Place Saint-Hubert, 59000 Lille, France. info@envisan.com

2. Grand Port Autonome de Dunkerque (GPMD), Terre-plein Guillain, BP 6534, Dunkerque, 59386 France. pgregoire@portdedunkerque.fr

3. Direction Technique COLAS Nord Picardie, 197 rue du 8 mai 1945 , F 59491 Villeneuve d'Ascq, France. christophe.priez@colas-np.com

4. Direction Technique COLAS Routes France, 4 rue Jean Mermoz- Bâtiment A, F 78772 Magny les Hameaux, France.

didier.desmoulin@colas.com

5. Directeur Marketing et Innovation Ciments Calcia et Unibeton, Les technodes, 78931 Guerville, France.

dguglielmetti@ciments-calcia.fr

\section{Résumé :}

Le Port de Dunkerque est en charge du dragage d'entretien et de la régulation des flux maritimes et donc du maintien de l'accessibilité des chenaux et darses situées dans le périmètre du Grand Port Maritime de Dunkerque (GPMD). Les résultats d'études de la qualité des sédiments ont indiqué des degrés variables de contamination, et en outre, les niveaux seuils "GEODE" officiels de la règlementation française ont été dépassés vis-àvis de certains métaux lourds. Sur la base de cette analyse, l'autorité de délivrance des permis a interdit la simple évacuation en eaux libres des sédiments dans la Mer du Nord. De ce fait, le GPMD a saisi l'occasion pour investir dans une nouvelle installation de traitement de 6 hectares et dans un large programme de valorisation des sédiments contaminés. Les sédiments dragués par voie hydraulique sont déshydratés par lagunage actif sur cette pate-forme, puis valorisé et orientés vers 3 filières. Ces 5 dernières années, avec ce programme, le GPMD a valorisé plus de $300000 \mathrm{~m}^{3}$ de sédiments contaminés retirés de ses darses au Port Est. Récemment, le Port a investi dans deux projets innovants de démonstration grandeur nature visant le réemploi utile des sédiments contaminés :

- Le premier projet a été mis en œuvre entre 2009 et 2013 : Un éco-modelé paysager de $500 \mathrm{~m}$ le long du Canal des Dunes, au sein de la zone portuaire. 


\section{Thème 8 - Gestion et valorisation des sédiments marins}

- Le deuxième projet a été mis en œuvre en 2012 : des sédiments contaminés ont été valorisés dans une nouvelle route, de 700 mètres de long, située dans le port de Dunkerque (Route Freycinet 12). Ce projet a compris l'essai préliminaire du mélange de sédiments en vue d'obtenir une qualité équivalente à celle des travaux de voirie classiques (en collaboration avec la société COLAS).

Le troisième projet a été réalisé durant l'été 2013 : il s'agit de la fabrication de 110 blocs de parement de brise-lames, au volume de 4 et $6 \mathrm{~m}^{3}$, recourant à une quantité maximum de sédiments de réemploi. Ces 4 prochaines années, 2 lieux d'approvisionnement sont prévus pour pouvoir effectuer les travaux de réparation sur le corps des digues. Fin septembre 2013, 4 blocs ont déjà été placés sur la Digue des Huttes à Dunkerque (en collaboration avec CTG).

Ces trois projets ont tous été exécutés par Envisan, choisi par le GPMD en raison de sa large connaissance du traitement des sédiments contaminés et du savoir-faire du Jan De Nul Group dans les secteurs du génie civil, des travaux maritimes et du dragage. La valorisation des matériaux dragués est actuellement en passe d'obtenir un cadre légal en France.

Mots-clés : Matériau dragué, Valorisation, Sédiments contaminés, Construction de digue, Béton, Technique routière, Sédiments non-immergeables.

\section{Introduction}

Le Grand Port Maritime de Dunkerque (GPMD) est situé sur la côte de la Mer du Nord et dispose d'un littoral de plus de $15 \mathrm{~km}$ de long. C'est le troisième plus grand port côtier de France, proche de la ligne de séparation du trafic traversant le détroit du Pas de Calais, sur la route maritime la plus dense du monde avec pas moins de 600 bateaux par jour. L'accumulation de sédiments entraîne la réduction du tirant d'eau nécessaire pour la navigation des cargos et bateaux-citernes de haute capacité ; par conséquent, les chenaux (sédiments sableux), avant-ports (sédiments très sableux) et darses (sédiments fins contaminés) doivent être dragués en permanence.

Le sable non-contaminé est stocké et commercialisé pour les remblais et les matériaux de construction. Durant des années, les vases et sédiments étaient clappés en mer, mais l'Arrêté Ministériel du 14 juin 2000 (JORF, 2000) impose à présent une gestion plus rigoureuse des sédiments, et interdit ce déversement pour les sédiments contaminés, qui doivent, depuis cette date, transiter à terre dans des sites sécurisés. Le GPMD, soucieux du respect de l'environnement, a ainsi choisi d'investir dans la construction d'un centre de traitement de ses sédiments dragués du port afin de ne plus rejeter ses sédiments contaminés, de niveau GEODE > N1 à la mer.

Le port de Dunkerque a estimé en 2000 que $500000 \mathrm{~m}^{3}$ de sédiments du Port Est étaient trop contaminés (principalement métaux lourds et HAP) pour être clappés en mer. C’est pourquoi, le port de Dunkerque a investi dans un large programme de valorisation de ses matériaux. 


\section{XIII ${ }^{\text {èmes }}$ Journées Nationales Génie Côtier - Génie Civil \\ Dunkerque, 2-4 juillet 2014}

\section{Caractéristiques des sédiments}

Les sédiments contaminés dragués ces 5 dernières années dans le cadre de ce programme proviennent tous de la partie est du port de Dunkerque. Leur granulométrie est (très) fine, leur teneur en eau élevée et leur teneur en argile variable (tableau 1). D’après le GTR (Guide Technique Routier) (LCPC \& SETRA, 1992), leur comportement géotechnique est comparable à celui de sols A1 ou de sols A3 pour les plus argileux. Les sédiments présentent souvent une grande teneur en matière organique, ce qui peut interférer avec l'ajout de liants hydrauliques. Par conséquent, leur classification géotechnique selon le GTR est F11 ou F12 (LCPC \& SETRA, 1992).

Tableau 1. Caractéristiques des sédiments.

\begin{tabular}{ll}
\hline Matière sèche (MS) & $45-50,5 \%$ \\
Matière organique (MO) & $9,7-12,3 \%$ \\
Teneur en argile $<2 \mu \mathrm{m}$ & $17-27 \%$ \\
Teneur en sable $>63 \mu \mathrm{m}$ & $35-15 \%$ \\
\hline
\end{tabular}

\section{Valorisation de sédiments contaminés non-immergeables dans un éco-modelé paysager}

De 2009 à 2013, chaque année, le volume total de $20000 \mathrm{~m}^{3}$ de sédiments contaminés a été valorisé après lagunage actif dans un éco-modelé paysager de 500 mètres érigée entre le "Canal des Dunes" et l'usine Polychim sur l'emprise du GPMD. Ce projet a inclus les travaux de construction civile, le recouvrement de terres végétales et la plantation d'espèces végétales spécifiques sur les talus. Des piézomètres ont été forés afin de contrôler le non impact de l'éco-modelé sur la qualité de la nappe aquifère sousjacente.

La conception de l'ouvrage prévoit une largeur variable à sa base ainsi qu'une pente variant entre 12/4 et 6/4. La hauteur varie de 5 à 7 mètres au-dessus du sol naturel. La conception finale de la digue ayant été établie par les Autorités portuaires, le taux de matière sèche à atteindre après lagunage $( \pm 70 \%)$ était déterminant pour la stabilité de la digue (les caractéristiques géotechniques des sédiments étant fixées). Le logiciel "DSheet piling" a été utilisée pour calculer la stabilité de la digue, en utilisant la cohésion et l'angle de friction interne mesurés lors d'un essai CU triaxial effectué sur le matériau laguné de Dunkerque après un compactage Proctor ( $\left.\mathrm{c}=11 \mathrm{kPa}, \mathrm{Phi}=22.7^{\circ}\right)$. 


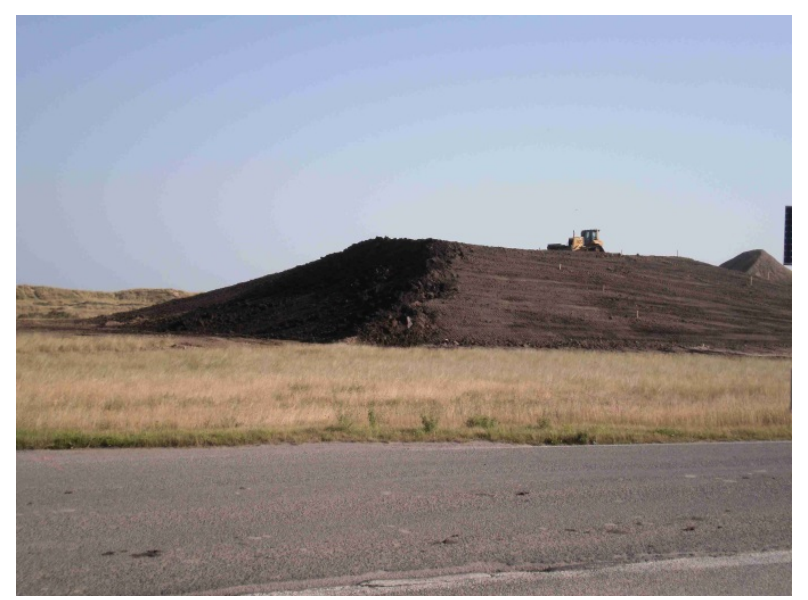

Figure 1. Eco-modelé paysager dont le cour est constitué de sédiments.

Une fois le cœur construit et recouvert de $25 \mathrm{~cm}$ de terre végétale, les espèces végétales endémiques suivantes ont été plantées sur ses pentes :

- 20\% de saule rampant (330 p.) (lat. Salix repens ss-esp. arenaria)

- 20\% de sureau noir (333 p.) (lat. Sambucus nigra)

- 50\% d'argousier (820 p.)(lat. Hipphophae rhamnoides)

- 10\% d'érable champêtre (165 p.) (lat. Acer campestre)

Pendant la période de croissance, les plantes ont subi des circonstances extrêmes. Un vent très fort et un temps très sec. Si bien que certaines espèces n’ont pas survécu, certaines étant plus sensibles que d'autres (ex. saule rampant contre argousier). Lors des campagnes, seules les espèces argousier et érable champêtre (80\%/20\%) ont alors été plantées.

L’épaisseur de la couverture de terres végétales a été contrôlée régulièrement afin d'étudier les effets de l'érosion du vent et du ruissellement (par levée topographique de l'éco-modelé paysager et vérification ponctuellement par des puits d’inspection). Aucune dégradation de cette couche n’a été observée. Toutefois, une clôture a été installée autour de l’ouvrage afin de prévenir tout endommagement de la terre végétale des pentes qui pourrait-être causé par la circulation de vélomoteurs et de quads.

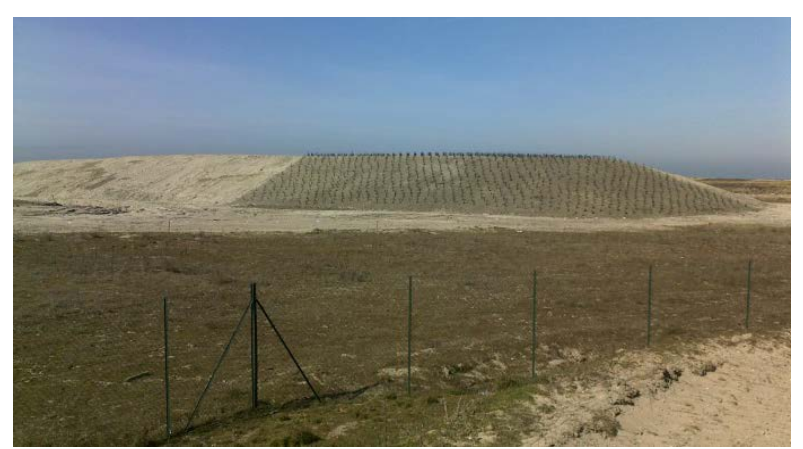

Figure 2. Eco-modelé paysager après plantation de végétation (1 $1^{e}$ saison). 


\section{XIII ${ }^{\text {èmes }}$ Journées Nationales Génie Côtier - Génie Civil \\ Dunkerque, 2-4 juillet 2014}

\section{Valorisation de sédiments contaminés non-immergeables dans la sous-couche et la couche de base de la structure en vue de la route "Freycinet 12".}

Les sédiments contaminés du Port de Dunkerque ont commencé à être réutilisés dans des structures routières dès 2002, date à laquelle le Port a débuté sa coopération avec l'Ecole des Mines de Douai et d'autres partenaires, comme la société COLAS en vue d'élaborer des matériaux alternatifs destinés aux couches stabilisées inférieures de voiries.

Après la première phase du projet, constituée d'essais en laboratoire, en 2005, un essai pilote a été exécuté sur une parcelle de $300 \mathrm{~m}^{2}$, où a été construite une route de 50 mètres de long recourant à des sédiments traités. L’objectif était de vérifier la faisabilité technique du processus dans son ensemble, qui comprenait les points suivants :

- Application d'une couche de sédiment ainsi qu'un apport de sable D1 (sable de dragage) pour être en conformité à l'étude réalisée préalablement en laboratoire et permettre la traficabilité du futur mélange.

- La recherche de l'optimisation géotechnique afin de concevoir un matériau routier ne peux en aucun cas être assimilé à une dilution de la contamination des sédiments, car le sédiment seule ne pouvait être utilisé seule. Le besoin de rectifier la granularité a été confirmé dans le projet SETARMS (BOUTOUIL et al., 2013).

- Traitement à la chaux vive (1\% à 3\%) pour traiter la matière organique, et contribuer à préparer un mélange tolérant un liant hydraulique. L'application de la chaux réduit la teneur en eau, flocule les particules d'argile et abaisse le taux de matière organique (CFTR \& SETRA, 2007).

- Ensuite, application d'un liant hydraulique à un taux usuelle en technique routière (>6\%), les liants hydrauliques ont été développés au travers du partenariat HOLCIM/COLAS, et il a été démontré qu'il est possible d'obtenir un matériau traité au liant hydraulique élaboré de catégorie T2 ou T3 au moyen d'un mélange contenant jusqu’à 30\% de sédiments.

Protection de la route/parcelle pilote au moyen d'un enduit de cure. Les résistances mécaniques (Rit/Eit) présentées grâce au carottage réalisé 6 mois après la réalisation du plot d'essai ont confirmé les résultats des études en laboratoire, ce qui a également permis de valider la technique employée.

Lors de l'analyse en labo, la performance mécanique du mélange sable/sédiments traités était de catégorie T3 (S3 : ancienne catégorie), avec un module de rigidité assez faible (point rouge sur la figure 4:2000 Mpa à $3000 \mathrm{Mpa}$ ). La performance mécanique mesurée sur les carottes du tronçon de route pilote confirme l'obtention d'un matériau de catégorie T3 mais avec un module de rigidité plus élevé. 


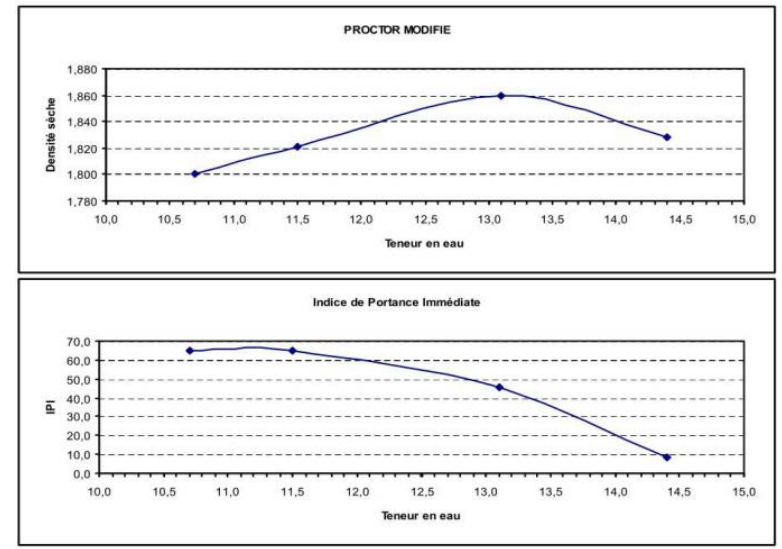

Figure 3. Courbe Proctor et courbe IPI des sédiments après traitement.

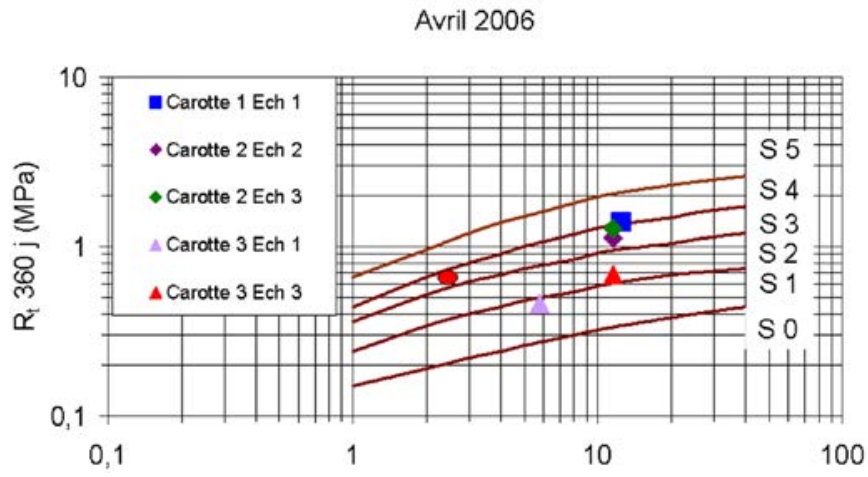

Figure 4. Résistance à la traction directe (Rt en Mpa) versus résultats du module élastique (Etd en GPa) des analyses de labo et des carottes de la route pilote de 2005 (point rouge).

L’expérience de 2005 a démontré qu’il était possible de formuler des matériaux routiers alternatifs incluant des sédiments.

En 2012, le Port de Dunkerque devait reconstruire la route "Route du Quai Freycinet 12" desservant Ruby Company, spécialisée dans le négoce de carburant. Il a donc choisi de mettre à l'épreuve la compétitivité d'une telle solution en passant un appel d'offre pour la construction de la Route Freycinet 12 permettant aux candidats de proposer une conception à base de sédiments. Le groupement ENVISAN - COLAS a finalement été sélectionné pour la construction de cette route grâce notamment à leur expertise en dragage, traitement de sédiments et techniques routières.

La structure routière a été conçue pour recevoir un trafic moyen de 100 poids lourds par jour sur une durée de vie de 15 ans, le dimensionnement a été réalisé en prenant en compte les performances mécaniques du sédiment traité, performances similaire à l'étude menée en 2005.

Dans l'appel d'offres, les services techniques du Port avaient conçues une structure usuellement utilisé en structure routière, à savoir $6 \mathrm{~cm}$ de Béton bitumineux sur $40 \mathrm{~cm}$ 


\section{XIII ${ }^{\text {èmes }}$ Journées Nationales Génie Côtier - Génie Civil \\ Dunkerque, 2-4 juillet 2014}

de grave laitier 0/20 sur une couche de forme de catégorie PF2 (composé principalement d'un sol sableux).

COLAS Nord Picardie a proposé la structure routière suivante, équivalente sur le plan mécanique : $5 \mathrm{~cm}$ de béton bitumeux à module élevé sur $6 \mathrm{~cm}$ d'EME (enrobé à module élevé) / $30 \mathrm{~cm}$ de matériau contenant les sédiments contaminés dragués et lagunés par Envisan sur le site des lagunes.

Cette solution emploie $1 \mathrm{~m}^{3}$ de sédiments pour $10 \mathrm{~m}^{2}$ de route. Ainsi, $450 \mathrm{~m}^{3}$ de sédiments secs, représentant $1800 \mathrm{~m}^{3}$ de sédiments dragués, ont été utilisés.

Une parcelle écologique a été installée sur une superficie de $100 \mathrm{~m}^{2}$ pour collecter les lixiviats de la route.

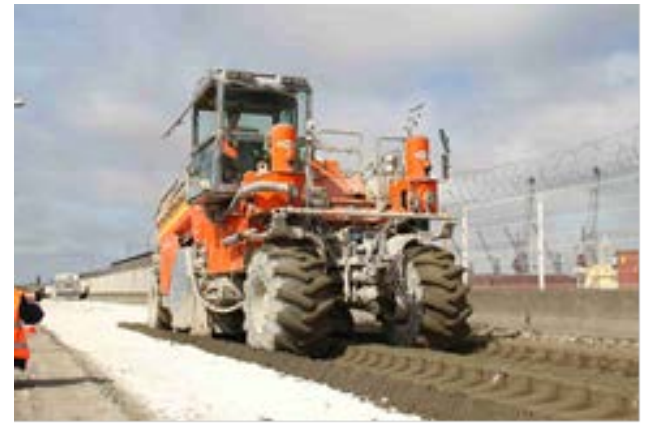

Figure 5. Traitement des sédiments de la route de Freycinet.

\subsection{Résultats géotechniques}

L'analyse en laboratoire de 2012 a donné des résultats moins optimistes que ceux de 2005. Toutefois, le mélange routier a atteint un niveau mécanique de classe T2, qui a été pris en compte dans l'élaboration de la structure routière. La résistance à la compression à court terme s'élève à $1,3 \mathrm{MPa}$ au bout de 7 jours, ce qui offre des conditions de trafic correctes pour l'exploitation normale sur site. À long terme, le module de rigidité dépasse $9000 \mathrm{MPa}$ et la résistance à la traction passe au-dessus de 0,6 Mpa.

Les carottes prélevées à 180 jours présentent une excellente cohésion du matériau, ainsi qu'un niveau de résistance à la traction égal à celui obtenu en labo.

L'écrasement mesuré 60 jours après la mise en œuvre (moyenne $<10 / 100^{\mathrm{e}} \mathrm{mm}$ ) est valable et montre l'efficacité de la couche à base de sédiments traités.

\subsection{Résultats du suivi environnemental}

Les sédiments employés dans la route ont été analysés suivant le guide méthodologique relatif à l'acceptabilité de matériaux alternatifs dans la construction de voiries (SETRA, 2011).

Cette analyse a confirmé l'efficacité du traitement par lagunage actif, car elle n’a révélé aucun problème vis-à-vis du taux de contaminants organiques (BTEX, PCB, THC, TOC et $\mathrm{PAH})$. 


\section{Thème 8 - Gestion et valorisation des sédiments marins}

En vertu de ce guide et de la législation d'application en France, les résultats des essais de lixiviation selon NF EN 12457-2 réalisés sur les sédiments bruts ont mis en évidence que certains seuils comme la fraction soluble, les sulfates et le chlorure présents dans les sédiments étaient dépassés.

Les tests de lixiviation selon NF EN 12457-2 effectués en partenariat avec Holcim sur les mélanges traités en labo et sur les carottes issues du chantier ont montré que les résultats étaient inférieurs aux seuils des déchets inertes.

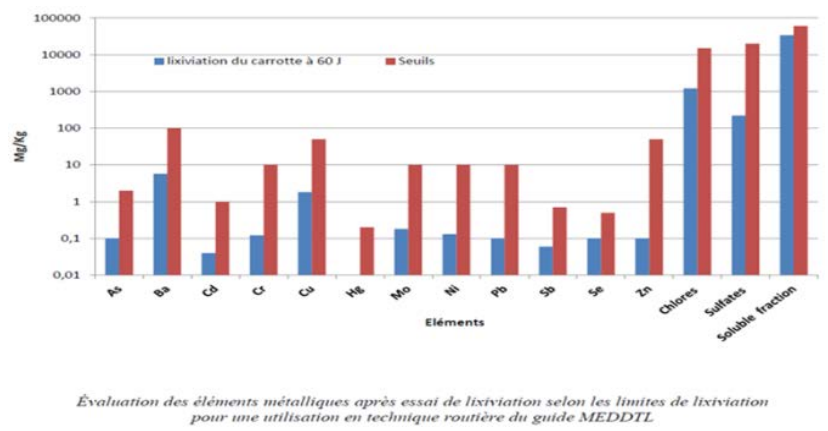

Figure 6. Résultats des essais de lixiviation sur les carottes après 60 jours (bleu) par rapport aux limites (rouge).

L'analyse des lixiviats sur la parcelle a confirmé le blocage des fractions solubles grâce au traitement au moyen d'un liant hydraulique d'inertage, avec des analyses approfondies du niveau seuil A1 des déchets de faible activité de l'eau.

Depuis la fin des travaux, les lixiviats des eaux souterraines, de la darse toute proche le long de la Route du Quai Freycinet 12 et du piézomètre ont été analysés tous les mois au cours de la première année et chaque semestre les 2 années suivantes. Un an après les travaux, aucun changement notable n'avait été décelé dans ces trois points de surveillance.

\section{Valorisation de sédiments contaminés dans des blocs brise-lames en béton}

Au printemps 2013, le Port de Dunkerque a attribué le projet de fabrication de 110 blocs de brise-lames (de 4 et $6 \mathrm{~m}^{3}$ de volume) à Envisan. Ces blocs contiennent des sédiments contaminés précédemment dragués dans le chenal de Broucqaire et dans la Darse 6. Avant d'être valorisés, en remplaçant partiellement la fraction de sable du béton par les sédiments, les sédiments contaminés ont été lagunés et prétraités avec $4 \%$ d’Alipré ${ }^{\circledR}$ (figure 7) sur le site des lagunes. Le béton a été produit dans la centrale à béton d'Unibéton, sur base de la formulation transmise par Italcementi. Dans les faits, la fabrication des blocs et leur placement sur le brise-lames de la section est du port de Dunkerque (Digue des Huttes) ont été exécutés en 2 mois.

Les sédiments contaminés non-immergeables (tableau 1) présentent le plus souvent une texture fine, une teneur en sulfates assez élevée et/ou une granularité bien répartie, ce 


\section{XIII ${ }^{\text {èmes }}$ Journées Nationales Génie Côtier - Génie Civil \\ Dunkerque, 2-4 juillet 2014}

qui en fait par définition un matériau pas facile à réutiliser dans le béton tel quel. En effet, les sédiments à grains fins ont pour caractéristiques une haute capacité de rétention d'eau, si bien que, sans prétraitement, ils obstruent les circuits d'alimentation traditionnels des centrales à béton. La solution à ce problème, mise au point et brevetée par le groupe Italcementi, consiste à prétraiter les sédiments lagunés au moyen d'ALIPRE ${ }^{\circledR}$. Ce liant à base de clinker calcium sulfo-alumineux (CSA) est fondamentalement un clinker sulfo-alumineux-bélite, dont les phases principales sont : calcium sulfo-alumineux, bélite et calcium sulfate. L'une de ses différences par rapport au ciment Portland classique est la formation d'ettringite primaire pendant l'hydratation, quand il est mélangé aux sédiments. Cette ettringite non seulement assèche le sédiment (en capturant l'eau dans les cristaux d'ettringite nouvellement formés), mais modifie aussi fortement la consistance des sédiments dans la centrale à béton.Après ce traitement, les sédiments ont une structure plus sableuse (d50 après traitement aux environs de $3 \mathrm{~mm}$, d50 initial $0.05 \mathrm{~mm}$ ), ce qui est très positif pour la suite de leur traitement.

Suite à l'échantillonnage de sédiments extraits des bassins de lagunage en décembre 2012, une vaste campagne d'analyses a été menée en laboratoire en vue d'ajuster la phase de prétraitement et la formulation du béton (analyses de labo réalisées par l'équipe R\&D d'Italcementi au CTG). En 2010 déjà, Italcementi s'était forgé une expérience approfondie de la valorisation des sédiments de Dunkerque dans le béton grâce à une campagne élaborée d'essais en laboratoire et à la fabrication pilote de 6 blocs de $4 \mathrm{~m}^{3}$ réalisés chacun selon une formulation de béton différente. La formulation de 2010 a été adaptée en tenant compte des caractéristiques géotechniques spécifiques des sédiments collectés en 2012.

En juillet 2013, des essais pilotes ont été menés dans le centre de traitement de terres et de sédiments d'Envisan situé à Gand, Belgique, sur des sédiments de granularité comparable.

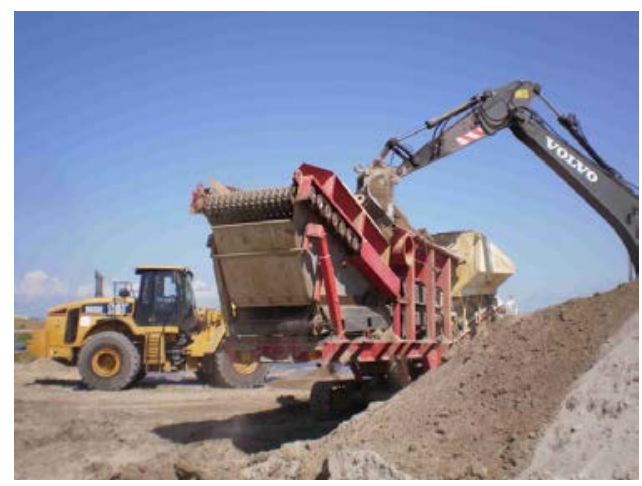

Figure 7. Prétraitement sur place de sédiments lagunés au moyen de l'additif CSA. 


\section{Thème 8 - Gestion et valorisation des sédiments marins}

Au cours de cet essai pilote, le dosage CSA selon la teneur en eau et la granularité des sédiments, de même que la granulation des agrégats de sédiments après traitement, ont été validés et optimisés. Sur la base de l'expérience acquise grâce aux essais pilote d'échelle 1, le traitement intégral des sédiments a été exécuté début août 2013, et ce, au moyen d'une installation mobile. Il s'est composé d'un criblage des sédiments avant leur mélange avec $4 \%$ de liant CSA.

Par la suite, une production test recourant aux sédiments traités a été effectuée à la centrale à béton d'Unibéton à Calais. Celle-ci a consisté en la fabrication de $3 \mathrm{~m}^{3}$ de béton employant les sédiments comme substitut partiel du sable. Les autres composants du béton fabriqué étant du sable, du gravier et du ciment utilisé pour la fabrication de béton classique. Les paramètres critiques évalués lors de cette production test étaient l'énergie de malaxage, l’ouvrabilité et la résistance à la compression du béton. Tous les tests s'étant avérés satisfaisants, la fabrication définitive des 110 blocs de béton a été lancée le 27 août, pour se terminer 12 jours plus tard. Les blocs de 4 et $6 \mathrm{~m}^{3}$ ne sont pas armés ; seul un crochet est prévu pour faciliter leur positionnement sur les brises-lame.

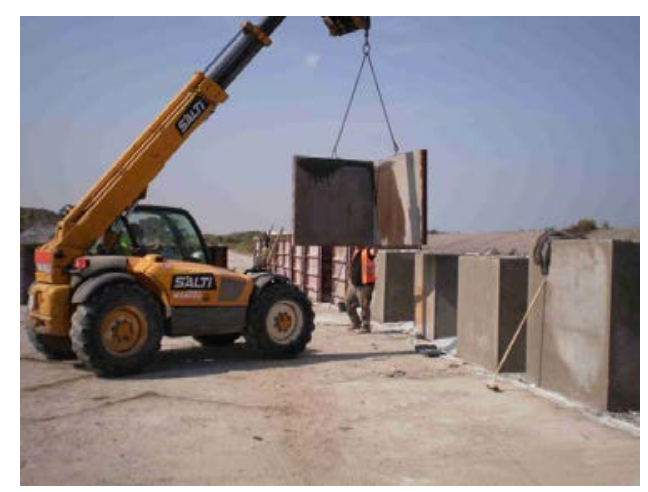

Figure 8. Démoulage des blocs sur place.

La résistance à la compression obtenue pour le béton sur le terrain était nettement supérieure au seuil requis de $30 \mathrm{MPa}$. Au bout de 28 jours, la résistance à la compression obtenue sur tous les échantillons cylindriques était supérieure à $39 \mathrm{MPa}$ (un béton C30/37 était exigé). En moyenne, les blocs en béton ont intégré entre 12 et $20 \%$ de sédiments, ce qui correspond grosso modo à $0,5 \mathrm{~m}^{3}$ de sédiments (in situ/avant dragage) par $\mathrm{m}^{3}$ de béton.

Un suivi de l'impact environnemental est envisagé pendant 1 an après la fin des travaux. Depuis la fin des travaux, une analyse des lixiviats dans les piézomètres, installés sur le site de stockage des blocs béton, est envisagée tout de suite après les travaux et chaque semestre pendant 1 an après la fin des travaux. 


\section{XIII ${ }^{\text {èmes }}$ Journées Nationales Génie Côtier - Génie Civil \\ Dunkerque, 2-4 juillet 2014}

Tableau 2. Critères de l'appel d'offres pour le béton.

Critères techniques de la soumission pour le béton

Résistance du béton BHCA C30/37 (béton Hors Champ d'Application, donc non soumis à la norme

béton EN206-1)

Consistance : $S 4$ (essai d'affaissement)

Béton exposé à l'eau de mer

Ciment conforme à la norme NF P 15317

Gravier conforme à la norme NF P 18545 et $D=\max .20 \mathrm{~mm}$

Facteur W/C maximum 0,55

Additifs conformes à NF EN 194-2

Eau selon NF EN 1008

Quantité de sédiments dans le béton : entre 12,5 et 20\%

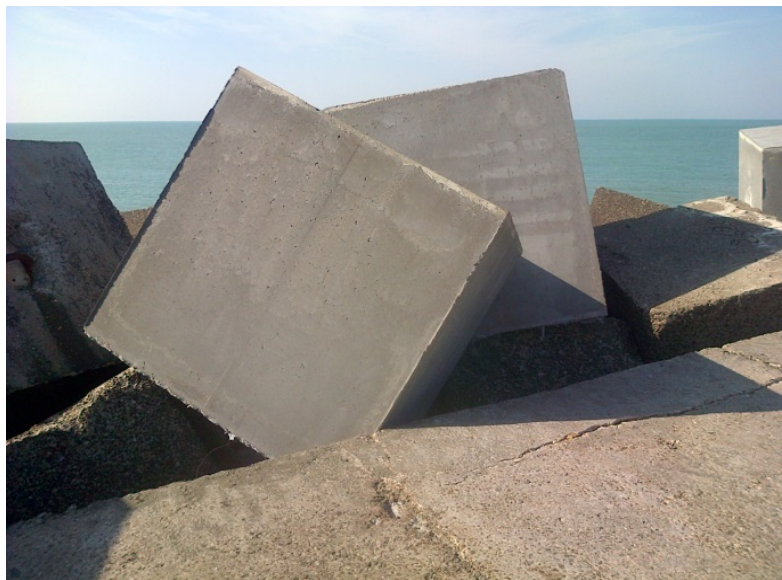

Figure 9. Premiers blocs de béton contenant des sédiments contaminés sur la Digue des Huttes à Dunkerque (septembre 2013).

\section{Conclusions}

Ces 5 dernières années, le Grand Port Maritime de Dunkerque (GPMD) a investi dans la mise en place de solutions innovantes et très opérationnelles pour répondre à son besoin impératif de filière de gestion à terre pour ses matériaux de dragage non immergeables. Cet investissement s'est traduit par la construction d'un centre de traitement de sédiment et la réalisation de 3 grands projets de travaux qui ont permis de valoriser près de la moitié du gisement de sédiments non-immergeables évalué au début des années 2000.

L'éco-modelé paysager du canal des dunes est ainsi composé à $92 \%$ de sédiments nonimmergeables, la route Freyssinet 12 est composée de près de 25\% de sédiments nonimmergeables alors que les blocs bétons en contiennent de l'ordre de $13 \%$. 
Le Port a ainsi démontré avec ses partenaires et prestataires tels qu'Envisan, Colas ou Italcimenti la faisabilité technique, administrative de réemployer les sédiments nonimmergeables dans des conditions environnementales acceptables et contrôlées.

Les matériaux non-immergeables issus du dragage des darses du Port est du GPMD jusqu'ici qualifiés par défaut, de déchets, du fait de leur dépôt à terre, ont ainsi conquit et acquis une utilité leur conférents un nouveau statut, à savoir celui de produit constitutif d'ouvrage utile et utilisé.

Cette démarche est rendue possible grâce à la parfaite connaissance du matériau dragué tant du point de vue chimique que géotechnique ou biologique mais aussi des avantages et inconvénients des techniques de dragage et de traitement des sédiments.

Cette expérience est exemplaire en France par son ampleur et sa singularité et ne devrait pas manquer de permettre d'assoir la définition d'une règlementation détaillée autorisant la valorisation de ces matériaux à grande échelle. Son existence même stricte offrirait un cadre pour le développement de solutions durables permettant de résoudre les problèmes de gestion des sédiments des Ports et voies navigables françaises.

\author{
Abréviations \\ BTEX : acronyme de benzène, toluène, éthylbenzène et xylène \\ PCB : polychlorobiphényl \\ HCT : hydrocarbures totaux \\ COT : carbone organique total \\ HAP : hydrocarbures polycycliques \\ CU triaxial : essai triaxial consolidé, non drainé
}

\title{
7. Références
}

JORF -Journal Officiel de la République Française- (2000). Arrêté du 14 juin 2000 relatif aux niveaux de référence à prendre en compte lors d'une analyse de sédiments marins ou estuariens présents en milieu naturel ou portuaire. JO n ${ }^{\circ} 184$ du 10 août 2000, texte $n^{\circ}$ 24, pp 12415-12416.

BOUTOUIL M., LE GUERN M., SAUSSAYE L., MAHERZI W. (2013). Sédiments de dragage traités aux liants hydrauliques- Evolution du comportement mécanique à court et long termes. Recyclage et valorisation, $n^{\circ} 41$ spécial juin 2013.

CFTR, SETRA (2007). Guide technique Traitement des sols à la chaux et/ou aux liants hydrauliques- Application à la réalisation des assises de chaussées.

LCPC, SETRA (1992). Guide Technique Réalisation des remblais et des couches de forme.

SETRA (2011). Guide méthodologique: Acceptabilité de matériaux alternatifs en technique routière-Evaluation environnementale. 